\title{
Italique
}

Poésie italienne de la Renaissance

I| 1998

Varia

\section{Rinaldo Corso e il "Canzoniere" di Vittoria Colonna}

Monica Bianco

\section{(2) OpenEdition}

Journals

Edizione digitale

URL: http://journals.openedition.org/italique/64

DOI: $10.4000 /$ italique.64

ISSN: 1663-4438

\section{Editore}

Librairie Droz

\section{Edizione cartacea}

Data di pubblicazione: 1 giugno 1998

Paginazione: $35-45$

ISBN: 2-600-00239-1

ISSN: 1423-3983

Notizia bibliografica digitale

Monica Bianco, «Rinaldo Corso e il "Canzoniere" di Vittoria Colonna », Italique [Online], I 1 1998, online dal 14 septembre 2009, consultato il 01 mai 2019. URL : http://journals.openedition.org/italique/64 ; DOI : 10.4000/italique.64

(c) Tous droits réservés 
MONICA BIANCO

RINALDO CORSO E IL “CANZONIERE”

DI VITTORIA COLONNA 


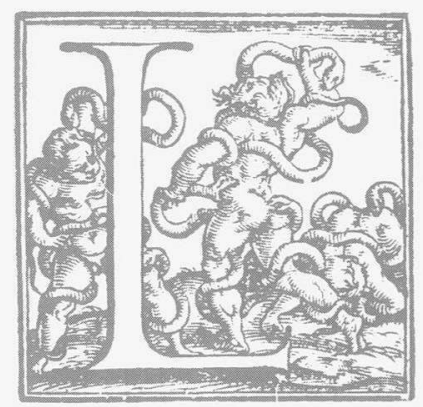

'Edizione de Le volgari opere del Petrarcha con la espositione di Alessandro Vellutello da Lucca, uscita a Venezia per i torchi dei fratelli da Sabbio nel 1525, era destinata ad aprire un capitolo nuovo nella storia dell'esegesi petrarchesca. Muovendo dall'assunto che le indicazioni cronologiche disseminate nel Canzoniere ne rispecchiassero l'effettivo ordine di composizione, il Vellutello contestava la legittimità dellordinamento dell'aldina del 1501 - il cui testo si dichiarava "tolto con sommissima diligenza dallo scritto di mano medesima del poeta, havuto da messer Pietro Bembo» - instaurando una nuova progressione dei componimenti ispirata insieme alla diacronia "interna" e ai dati della biografia petrarchesca. Dalle due parti canoniche - rime in vita e in morte di Laura - rimaneva così escluso un manipolo di testi di corrispondenza relegato a formare una terza parte, che si giustapponeva alle precedenti quasi come un'appendice di estravaganti. ${ }^{2}$ L'esperimento del Vellutello aveva aperto una questione che sarebbe stata ineludibile per i successivi editori e commentatori del Canzoniere. E tutti, almeno fino agli anni' 40 , sia che tentassero nuove strade, come Fausto da Longiano, che divise $i$ testi in sonetti e canzoni sull'esempio degli antichi canzonieri due-trecenteschi, sia che tornassero all'ordinamento dell'aldina, come il Gesualdo o il Daniello, avvertirono la necessità di premettere alle loro fatiche una nota specifica sulla disposizione delle rime. ${ }^{3}$ Se l' "eterodossia" del nuovo ordinamento, che smentiva insieme ad Aldo il Bembo, era costata al Vellutello l'aperta ostilità dei letterati vicini al futuro cardinale, la sua iniziativa editoriale non aveva mancato di riscuotere altrove significativi consensi. Nel 1554 usciva a Venezia per le cure di Girolamo Ruscelli un'edizione non commentata del Canzoniere nella quale $i$ testi, pure presentati nella lezione di Aldo, erano disposti secondo lordine del Vellutello. Nella lettera ai lettori, dando ragione della scelta operata, il Ruscelli dichiarava: "Ancor l'ordine del Vellutello potesse migliorarsi, come bene avvertisce messer Rinaldo Corso in una compendiosa e utilissima esposition sua, noi (...) non habbiamo voluto alterar questo che fin qui è andato attorno, essendo nel vero in quanto al tutto o alla maggior parte migliore di tutti gli altri». ${ }^{4}$

Il Rinaldo Corso chiamato in causa dal Ruscelli viveva a Correggio, dove era nato nel 1525, e ivi svolgeva le funzioni di giudice e di priore del Collegio dei notai. Animatore della vita culturale cittadina, dopo aver partecipato assiduamente all'attività dell'accademia che si riuniva intorno 
a Veronica Gambara, ne aveva fondato una nuova, detta dei Filogariti. Era poeta ed autore di una grammatica, $i$ Fondamenti del parlar thoscano, stampata a Venezia presso Comin da Trino nel 1549.5 Quali modifiche il Corso ritenesse atte a perfezionare la disposizione del Vellutello non è possibile dire, dal momento che la sua "espositione» risulta a tutt'oggi irreperibile. Che l'esperimento del letterato lucchese l'avesse nel suo complesso convinto trova però conferma nell'opera con la quale egli, sedicenne, aveva scelto di presentarsi sulla scena letteraria: il commento alle rime di Vittoria Colonna.

L'annotazione alle rime amorose e spirituali della marchesana di Pescaraimpresa in quegli anni assolutamente innovativa, considerato che l'autrice era ancora vivente - fu compiuta a Correggio durante l'estate del 1541. Due anni dopo, nel 1543, lo stesso autore faceva uscire a Bologna, con dedica a Veronica Gambara, la sola seconda parte del commento, consacrata alle rime spirituali. ${ }^{6}$ L'opera completa vedeva la luce, dopo essere stata sottoposta ad una consistente rielaborazione, che non intaccava tuttavia l'ordinamento dei testi, a Venezia presso i Sessa solo nel 1558.

Delle sei raccolte di rime della Colonna stampate tra il 1538 e il $1540,{ }^{8}$ il Corso decise di usare come edizione di riferimento la piu recente, che si aumentava di dodici testi inediti, e la segui non solo nelle lezioni, ma in due casi financo nei refusi. Nessuna delle raccolte apparse aveva ottenuto il benestare della poetessa. Custode gelosa dei propri scritti, ${ }^{9}$ Vittoria Colonna non solo non curò mai uniedizione a stampa delle rime, ma non volle mai avallare quelle che uscirono. Per quanto perteneva all'ordine delle composizioni, l'indisponibilità di un'edizione riconducibile alla volontà d'autore poneva un problema analogo a quello sollevato dal Vellutello per il Canzoniere petrarchesco. Rinaldo Corso optò per la stessa soluzione di deciso intervento editoriale: l'opera del commentatore avrebbe avuto come necessaria premessa la "restituzione" di un ordine dei testi.

Nelle sei edizioni citate l'unica disposizione vigente era quella per generi metrici: un capitolo ternario (ma solo dal 1540), i sonetti, le canzoni e le stanze. ${ }^{10}$ All'interno del gruppo dei sonetti si era poi progressivamente delineata una separazione tra rime amorose e spirituali. Queste ultime, poste dapprima a fine volume, erano state spostate all'inizio nell'edizione del 1539 (uscita a Firenze, ma "ad instantia di Nicolò d'Aristotile detto il Zoppino da Ferraras) e delimitate dalla scritta "Finisse gli sonetti spirituali»; la loro presenza era li per la prima volta dichiarata nel frontespizio. Nella raccolta del 1540 lo Zoppino, avvertito di quanto le primizie spirituali della Colonna potessero sollecitare l'interesse dei lettori, aveva fatto in modo che anche la 
presentazione editoriale desse risalto alla nuova produzione. ${ }^{11}$ Il Corso radicalizzò il processo già in atto proponendo, secondo il modello petrarchesco, un canzoniere bipartito e, rovesciando la disposizione che trovava nella sua raccolta di riferimento, pospose le rime spirituali a quelle amorose. Non segui le orme del Vellutello fino a tripartire il canzoniere, ma raccolse comunque $i$ dodici sonetti di corrispondenza a formare un gruppetto compatto, collocandoli in coda ai sonetti amorosi. Che il Corso considerasse questa una sezione in qualche modo autonoma è testimoniato dalle righe che aprono il commento al secondo sonetto della prima parte, Le tante opre divine e il sacro impero: "Se io fossi, leggiadre donne, certo il presente sonetto essere scritto a monsignor Bembo, come da alcuni ho inteso, senza dubbio lontano da qui sotto altro ordine posto l'havrei; ma non havendo piu certezza che io m'habbia, qui l'ho posto, parendomi che al suo Sole [cioè a Ferdinando Francesco d'Avalos] convenientemente possa essere scritto". 12

All'interno delle due sezioni il Corso divise le rime secondo il metro, giustificando la scelta all'inizio della seconda parte del commento: "Sì come la prima parte habbiamo chiusa con le due canzoni per esser da $i$ sonetti differente maniera di dire, così in questa per essere differente la maniera de' terzetti dall'ordine de' sonetti questi habbiam posto innanzi». ${ }^{13}$

La ripartizione che ne deriva è la seguente: rime amorose 118 sonetti (106 amorosi e 12 corresponsivi) seguiti da due canzoni; rime spirituali 37 sonetti seguiti da un capitolo ternario.

Dei 167 componimenti contenuti nella raccolta del 1540 il Corso scelse di commentarne $157^{14}$ e questi "sparsi frammenti" dispose in modo da suggerire il percorso dell'anima di Vittoria Colonna dall'amore umano a quello per Dio. Quello che il Corso si propose di fare fu di "procurare, ai fini pratici, una piu facile leggibilità del testo, una sua fruizione in chiave essenzialmente narrativan, ${ }^{15}$ obiettivo chiaramente enunciato all'inizio della seconda parte del commento:

Sì come d'hora in hora nascono pensier nuovi e dopo lungo tempo alcuna volta quelli medesimi tornano, così diverse compositioni e doppo lungo spatio di tempo talhora le medesime sententie sotto differente velo di parole si dipingono, talmente che di rado avviene che ciascuna compositione per sé non istia senza havere da altra dipendenza e vana quasi dir possiamo la fatica di voler quelle congiungere. È tuttavia quasi maggior sodisfacimento di chi legge, poi che raccolte sono, trovarle per ordine poste e continuatamente dichiarate. Il che noi come nella prima parte et in questa ancora si può vedere habbiamo alquanto ne' sonetti osservato. ${ }^{16}$ 
Il criterio di accorpamento dei testi fu, come già in Vellutello, quello della pertinenza dei soggetti e dell'unità degli argomenti: le rime furono riunite in gruppi affini per tematica e i legami non solo tra $i$ diversi gruppi, ma tra $i$ singoli componimenti sottolineati nell'esegesi. Oltre alla frequenza delle formule di raccordo poste ad apertura di commento quali: «avendo nel precedente sonetto Vittoria nostra detto (...) ora in questo", "segue Vittoria nostra dicendo", "mostra similmente Vittoria nostra", "riprende Vittoria nostra quello che ha detto nel precedente sonetto", ${ }^{17}$ colpisce la fitta rete dei rimandi interni. Sempre attento alla coerenza del discorso, il Corso cerca, per quanto possibile, di appianare eventuali incongruenze col ricorso a formule di occupatio quali ad esempio: "non è Vittoria nostra, come ad alcuni forse parrebbe, contraria a quel che habbiamo noi detto nel precedente sonetto». ${ }^{18}$

La prima parte del canzoniere si apre con i sonetti Scrivo sol per sfogar l'interna doglia $e$ Le tante opre divine e 'l sacro impero' ${ }^{19}$ aventi come soggetto le ragioni della scrittura e il topos dell'ineffabilità. Ai canoni dell'exordium rispondono sia la captatio benevolentiae (I, 9-10) sia le dichiarazioni sul genere, sullo stile e sulla materia (II, 1-7). Con il successivo sonetto si ha l'initium narrationis e nel commento ad esso il Corso anticipa brevemente la parabola narrativa della prima parte: «Ha scritto questo sonetto Vittoria nostra volendoci, a confermatione di quanto ha detto ne precedenti sonetti, mostrare quanto fin dallo stesso nascimento era ordinato in cielo del suo consorte e quanto a lui furon propitie e larghe le buone stelle e successivamente dimostrarci, come andando più oltre vedremo, quanto ella di lui degnamente saccendesse, onde la giustissima cagione che ha a dolersi della sua morte aperta ne segua». ${ }^{20}$ Il sonetto III e quelli che seguono sono consacrati all'encomio di Ferdinando Francesco d'Avalos: di hii Vittoria celebra il giorno natale, nel quale gli furono elargite dalle stelle le più alte virtù (III-IV), ${ }^{21}$ dichiarandosi consapevole dell'inadeguatezza dei propri versi a paragone di esse (V-XI). ${ }^{22} \mathrm{Il}$ trait d'union con $i$ sonetti XII-XXX ${ }^{23} \dot{e}$ indicato dal Corso allinizio del commento a XII: "Havendoci ne primi sonetti assai bene dimostrato la marchesana nostra quanto al suo Sole fossero stati larghi i cieli, ora s'apparecchia a dimostrarne come egli bene adoperò quanto favore haveva dal cielo havutom. ${ }^{24}$ Con Occhi miei, oscurato è il nostro Sole $(X X X I),{ }^{25}$ che mutua l'incipit di una delle rime di compianto del Petrarca (Rvf. 275), hanno inizio $i$ sonetti del lutto: il cuore della poetessa è urna delle ceneri del consorte (XXXII, A1: 34) e vittima sacrificale (XXXIII, A1: 55). A tre testi che descrivono lo smarrimento provato da Vittoria in seguito alla morte del marito, incentrati sullimmagine della vita come mare in 
tempesta e dell'uomo come fragile barca $(X X X I V-X X X V I),{ }^{26}$ il Corso ne affianca sette $(X X X V I I I-X L I V)^{27}$ aventi per tema lo stato di prostrazione che impedisce alla poetessa di gioire delle cose belle, come il sole e la primavera, e a questi, in base alla considerazione che un dolore insostenibile, quale è quello per un uomo senza pari, pù̀ trovare pace solo nella morte, dodici (XLV-LVI ${ }^{28}$ nei quali Vittoria si mostra tentata da pensieri suicidi e confessa che seguirebbe con gioia l'esempio di Porzia se non la trattenesse il timore di perdere con la salvezza la possibilità di riunirsi per sempre al consorte in cielo. Con un certo stacco rispetto ai precedenti, sono collocati a questo punto due sonetti (LVII-LVIII) ${ }^{29}$ aventi in comune il tema dell'anniversario della morte dell'Avalos (rispettivamente il quarto e il settimo). Emerge qui con tutta evidenza come la strategia messa in opera dal Corso privilegi l'avvicinamento di gruppi di testi legati da affinità tematica rispetto ad una disposizione più o meno forzosamente cronologica. I successivi sonetti LIX-LXX ${ }^{30}$ sono accomunati dalle immagini della fiamma e del nodo d'amore. Il tema della memoria che "sol (...) nel dolor savviva" (LXXVIII, 8) e l'immagine dell'amato scolpita nel cuore legano tra loro $i$ sonetti $L X X I-L X X X,{ }^{31}$ ai quali sono fatti seguire tre testi (LXXXI-LXXXIII), ${ }^{32}$ nei quali Vittoria "ci dà a vedere che il suo Sole, essendo stato suo duce in terra, ora similmente è nel cielo». ${ }^{33}$ Vengono poi i sonetti su ciò che è di conforto al dolore di Vittoria: il rivolgere il pensiero al marito (LXXXIV-XCVIII), ${ }^{34}$ il sonno (XCIX-CII), ${ }^{35}$ la poesia (CIII, A1: 51), serie suggellata tuttavia da CIV (A1: 70) in cui la poetessa ribadisce linnestinguibilità del suo pianto. A conclusione dei testi più propriamente amorosi è posto S'io potessi sfrondar da l'empia e folta $(C V)$, di cui è evidenziata la funzione di ponte con le rime spirituali: "Cominciando già Vittoria nostra a sentir nel suo cuore una scintilla del divino amore che a sé la chiama, si scusa di non poter così tosto, come si converrebbe, dipartir la mente da' terreni pensieri per esservi già troppo invecchiata). ${ }^{36}$ Se '1 mio bel Sol e l'altre chiare stelle (CVI, A1: 83), in cui la Colonna accenna agli altri lutti familiari che l'hanno colpita, funge da anello di congiunzione tra $i$ sonetti amorosi e $i$ seguenti dodici di tipo epistolare (CVII a Giovanna d'Aragona, CVIII-CIX sulle vittorie di Carlo V, CX ad Alfonso d'Avalos, CXI a Pietro Bembo, CXII a Pompeo Colonna, CXIII in morte di Paolo Giovio, CXIV a Veronica Gambara, CXV-CXVIII a Francesco Maria Molza). ${ }^{37}$ Sono poste a conclusione le due canzoni Spirto gentil, che sei nel terzo giro, dell'Ariosto, ${ }^{38}$ ma piegata a lamento di Vittoria per la morte del marito, e Mentre la nave mia lunge dal porto ${ }^{39}$ in cui la poetessa paragona il suo dolore a quello di celebri eroine: Penelope, Laodamia, Arianna, Medea, Porzia. 
La seconda parte del canzoniere si apre con una serie di testi consacrati al tema della mutatio vitae. Nei primi due sonetti, Con la croce a gran passi ir vorrei dietro $e$ Tempo è pur ch' io con la precinta vesta, ${ }^{40}$ accomunati dalla matrice scritturale, l'intenzione è espressa prima parafrasando le parole di Gesù in Matteo XVI, 24 poi, secondo la parabola evangelica (Matteo, XXV, 1-13), con l'immagine della vergine saggia. Il rinnovarsi della materia ora fatta "spirituale" comporta una sorta di nuova protasi: nei sonetti III-V $V^{41}$ Vittoria invoca come numi tutelari non piu Apollo e le Muse, ma Dio e i cori angelici. A questo primo gruppo sono accostati dal Corso sette sonetti (VI-XII ${ }^{42}$ nei quali la poetessa, dubitando di poter condurre a termine il nuovo compito con le sole sue forze, chiede lintervento della Grazia divina. Segue un cospicuo manipolo di rime sulla Redenzione e sulla vita eterna (XIII-XXIII), ${ }^{43}$ al quale sono avvicinati cinque sonetti di meditazione sulla misericordia del Salvatore (XXIVXXVIII). ${ }^{44}$ Il fatto che gli uomini si mostrino indegni di essa al punto da muovere Dio all'ira collega ai precedenti Padre Noè, del cui buon seme piacque $(X X I X)$ sul diluvio, Le nostre colpe han mosso il tuo furore $(X X X)$ sugli orrori contemporanei, moderno diluvio, e Di vero lume abisso immenso e puro (XXXI) in cui Vittoria supplica il Signore di dare saggezza ai potenti. ${ }^{45}$ Non a caso la rivolta di Lucifero è l'argomento del secondo dei due seguenti sonetti sugli angeli (XXXII-XXXIII); 46 vi si contrappone in XXXIV-XXXV ${ }^{47}$ il tema della povertà ed umiltà di Cristo e di san Francesco. Il sonetto XXXVI (Donna secura, accesa e da l'errante, S1: 121) sulla Maddalena è esplicitamente collegato al successivo XXXVII (Quando, vostra mercè, quasi presente, S1: 50), sullesperienza mistica di Vittoria, attraverso il parallelo fra le due vicende "agiografiche": "Giudico io Vittoria nostra in questo sonetto con qualche divotissimo padre del venerabile Ordine dé poveri Cappuccini di san Francesco, di cui ella, non men che Maddalena già di Cristo, si mostra discepola o d'altro tale, ragionare». ${ }^{48}$ Il canzoniere si chiude col capitolo ternario Poi che 'l mio Sol d'eterni raggi cinto, ${ }^{49}$ nel quale la poetessa descrive una visione di Cristo crocifisso in gloria tra Maria e la Maddalena, sulla quale, dimentica di tutto, mantiene fisso lo sguardo. L'itinerarium Victoriae in Deum tenuto da Rinaldo Corso trova qui il suo suggello.

Monica Bianco 


\section{Rinaldo Corso e Vittoria Colonna}

1. L'affermazione, contenuta nel colophon, era ribadita nella postilla di "Aldo a gli lettori». Le cose volgari di messer Francesco Petrarcha, Venezia, Aldo Manuzio, 1501, c. B 3r. Sui rapporti tra Aldo e il Bembo si veda C. Dionisotti, Aldo Manuzio editore, in Id., Aldo Manuzio umanista editore, Milano, 1995, pp. 91-138.

2. Sul commento del Vellutello si veda G. Belloni, Laura tra Petrarca e Bembo. Studi sul commento umanistico-rinascimentale al "Canzoniere", Padova, 1992, pp. 58-95.

3. Si vedano le osservazioni di G. Gorni, Le forme primarie del testo poetico, in Id., Metrica e analisi letteraria, Bologna, 1993, pp. 115-18.

4. Il Petrarca nuovamente con la perfetta ortografia della lingua volgare corretto da Girolamo Ruscelli, Venezia, Plinio Pietrasanta, 1554, cc. * 5v.- * 6r.

5. Il Corso avrebbe abbracciato nel 1567 lo stato ecclesiastico, finendo la sua vita vescovo di Strongoli in Calabria probabilmente nel 1581. Per altre notizie su di lui si veda la voce curata da G. Romei nel Dizionario biografico degli italiani, XXIX, Roma, 1983, pp. 687-90.

6. Dichiaratione fatta sopra la seconda parte delle rime della divina Vittoria Colonna marchesana di Pescara da Rinaldo Corso, Bologna, Giambattista Faelli, 1543 (d'ora in poi D 1543). La data di composizione dell'opera è indicata dal Corso nella lettera di dedica a c. A 3r.

7. Tutte le rime della illustrissima et eccellentissima signora Vittoria Colonna marchesana di Pescara con l'espositione del signor Rinaldo Corso, Venezia, Giovambattista e Melchior Sessa, 1558 (d'ora in poi E 1558).

8. Alla princeps uscita a Parma nel 1538 erano seguite quattro edizioni nel 1539, una sola delle quali con indicazione di città e stampatore (Firenze, Zoppino), e una nel 1540. Si veda VITTORIA Colonna, Rime, a cura di A. Bullock, Bari, 1982, pp. 258-61. Il Bullock segnala (p. 261), siglandola Rime-1 1540 e quindi datandola a quell'anno, una stampa che è invece una diversa emissione dell'edizione siglata Rime 1542. Poichè il colophon degli esemplari di entrambe le emissioni reca la data 1542, l'edizione è da collocarsi in quell'anno.

9. A quanto sappiamo, in tre soli casi accettò che fossero trascritte sue rime per inviarle a persone di particolare riguardo: Francesco Della Torre, Margherita di Navarra e Michelangelo Buonarroti. Le tre raccolte sono state identificate, ma non mancano fondati dubbi nel secondo caso, con i seguenti manoscritti: Firenze, Biblioteca Nazionale Centrale, II, IX, 30; Firenze, Biblioteca Medicea Laurenziana, Ashburnham 1153; Città del Vaticano, Biblioteca Apostolica Vaticana, Vaticano Latino 11539.

10. Le stanze Quando miro la terra ornata e bella, attribuite dalle stampe alla Colonna, erano in realtà di Veronica Gambara. Cfr. Veronica Gambara, Le rime, a cura di A. Bullock, FirenzePerth, 1995, p. 129.

11. Il frontespizio reca due incisioni: una sul recto raffigurante una donna in preghiera davanti a un crocifisso e una sul verso raffigurante la crocifissione. Si veda C. Dionisotti, Appunti sul Bembo e su Vittoria Colonna, in Miscellanea Augusto Campana, I, Padova, 1981, pp. 281-82.

12. E 1558 , p. 7. Riprova della voce riportata dal Corso a proposito di questo sonetto, che nell'edizione critica fa parte di quelli spirituali (S1: 139), si ha nel manoscritto 2051 della Biblioteca Angelica di Roma, che ha la didascalia «al Bembon. Cfr. VitToria Colonna, Rime cit., p. 506 


\section{Monica Bianco}

13. E 1558 , p. 394.

14. Tre di essi non erano di Vittoria: Spirto gentil, che sei nel terzo giro di Ludovico Ariosto, attribuzione di cui il Corso mostra di essere al corrente (E 1558, p. 350), ma dato dalle stampe alla Colonna, essendo stato scritto dall'autore come lamento di Filiberta di Savoia per la morte del marito Giuliano de' Medici; Alta fiamma amorosa e ben nate alme di Francesco Maria Molza, che pure si trovava nelle stampe tra le rime della marchesana; Sio potessi sfrondar da l'empia e folta attribuito dal Bullock, contro tutta la tradizione, a Pompeo Colonna (cfr. A. Bullock, Vittoria Colonna e i lirici minori del Cinquecento: quattro secoli di attribuzioni contraddittorie, "GSLI", 157 (1980), pp. 389-91).

15. G. Gorni, Le forme primarie cit., p. 117.

16. E 1558 , pp. 393-94.

17. Sono le formule che a sua volta il Vellutello aveva usato per legare l'una all'altra le rime del Canzoniere: "havendo il poeta ne' due precedenti sonetti dimostrato (...) hora in questo», "seguita il poeta nel presente sonetto", "mostra pur ancor il poeta». Non a caso. La loro presenza si rivelava necessaria in due commenti nei quali l'esegesi ai singoli componimenti, giustificandone la nuova localizzazione, veniva a costituire la vera nota al testo.

18. E 1558 , p. 403.

19. A1: 1, S1: 139. Data la maggiore reperibilità dell'edizione critica, le rime saranno citate anche secondo la numerazione in essa adottata. Va tenuto però presente che E 1558 riporta molti testi in una lezione antecedente rispetto a quella dei due testimoni sui quali il Bullock ha basato detta edizione.

20. E 1558, p. 12.

21. A1: 16, A2: 23

22. A2: 28, A2: 19, A1: 74, A2: 31, A1: 75, A1: 24, A1: 71.

23. A1: 62, A2: 3, A2: 4, A1: 6, A1: 5, E: 24, A1: 42, A1: 12, A1: 77, A2: 21, A1: 57, A1: 49, A1: 43, A2: 33, A1: 39, A1: 17, A1: 8, A1: 50, A1: 4 .

24. E 1558 , p. 40.

25. A1: 15 .

26. A1: 28, A1: 9, A1: 53. La disposizione è a climax: in XXXIV l'immagine ricorre nell'ultima terzina, in XXXV il confronto tra passato felice e presente doloroso fa sì che il sonetto cominci $O$ che tranquillo mar, che placide onde, in XXXVI (Solco tra duri scogli e fieri venti) il mare in tempesta è presente sin nell'incipit.

27. A1: 32, A1: 21, A1: 11, A1: 19, A2: 12, A1: 13, A1: 33. Non esiste in E 1558 un sonetto numerato XXXVII; la numerazione esatta è ripristinata più avanti col sonetto LXXI bis.

28. A1: 22, A2: 18, A1: 44, A2: 43, A1: 26, A2: 8, A1: 78, A2: 6, A1: 56, A1: 64, A1: 72, A 1: 47 . 


\section{Rinaldo Corso e Vittoria Colonna}

29. $A 1: 59, A 2: 29$.

30. A1: 7, A2: 20, A1: 45, A1: 48, A1:23, A2:44, A1: 55, A2: 30, A1:29, A1: 10, A1: 31, A1: 66 .

31. A1: 62, A1: 61 (LXXI bis), A1: 27, A1: 3, A1: 80, A1: 85, A1: 18, A1: 30, A1: 2, A1: 58, A1: 65 .

32. A1: $63, \mathrm{~A} 1: 76, \mathrm{~A} 2: 39$.

33. E 1558 , p. 233.

34. A1: 52, A2: 32, A2: 36, A1: 60, A1: 37, A1: 38, A1: 41, A2: 14, S2: 35, A2: 13, A1: 36, A1: 25, A1: 40, A2: 25, A1: 54 .

35. A1: 69, A1: 20, A1: 14, A1: 68 .

36. E 1558 , p. 303.

37. E: 10, E: 26, E: 25, E: 2, E: 14, E: 5, E: 1, E: 13, E: 17, A1: 88, Alta fiamma amorosa e ben nate alme, E: 18 .

38. Cfr. nota 14 .

39. A1: 89.

40. S1: $5, \mathrm{~S} 1: 8$.

41. S1: $1, S 1: 2, S 2: 1$.

42. S1: 52, S1: 10, S1: 88, S1: 84, S1: 57, S1: 53, S1: 12.

43. S1: 92, S2: 22, S1: 100, S1: 54, S1: 114, S1: 115, S1: 83, S2: 5, S1: 24, S1: 6, S1: 18.

44. S1: 51, S1: 12, S1: 13, S2: 11, S1: 55.

45. S1: 111, S1: 99, S1: 93.

46. S1: 7, S1: 132 .

47. S1: $98,51: 124$.

48. E 1558 , p. 471. Il "divotissimo padre» nella prima redazione, conservata da D 1543 , era Bernardino Ochino.

49. S2: 36 . 